\title{
CORRECTION AND SUPPLEMENTS TO "SCATTERING MATRICES AND SCATTERING GEODESICS OF LOCALLY SYMMETRIC SPACES"
}

\author{
BY LIZHEN JI AND MACIEJ ZWORSKI
}

\begin{abstract}
This note corrects and complements our paper entitled "Scattering matrices and scattering geodesics of locally symmetric spaces" (Ann. Sci. Éc. Norm. Sup. 34 (2001) 441-469).

(ㄱ) 2002 Éditions scientifiques et médicales Elsevier SAS
\end{abstract}

RÉSUMÉ. - Nous apportons des corrections et des compléments à notre article intitulé "Scattering matrices and scattering geodesics of locally symmetric spaces" paru dans les Ann. Sci. Éc. Norm. Sup. 34 (2001) 441-469.

○ 2002 Éditions scientifiques et médicales Elsevier SAS

\section{Introduction}

In his review of [4] in [2], Hoffmann pointed out the misleading comments after Definition 2.7 in [4] and a mistake in the proof of Proposition 2.10 in bounding the sojourn time in terms of the Langlands decomposition. He also pointed out a generalization in [3, Theorem 7.1] to $\mathbb{Q}$-rank 1 spaces of Guillemin's formula in [1] expressing the scattering matrices in terms of the sojourn times of scattering geodesics. The purposes of this note are to clarify the definition of the sojourn time and to relate it to the Bruhat decomposition, and to give a proof in Proposition 2.3 of discreteness of the sojourn times in Proposition 2.10 in [4] communicated to us by Hoffmann. The connection between the Bruhat decomposition and the sojourn time in Propositions 2.1 and 2.2 was claimed in [3, p. 120] and is of independent interest.

\section{The sojourn time and the Bruhat decomposition}

Recall from [4] that $\Gamma \backslash X$ is a $\mathbb{Q}$-rank one locally symmetric space, and that there is a bijection between the set of ends of $\Gamma \backslash X$ and the set of $\Gamma$-conjugacy classes of proper rational parabolic subgroups of G. Let $P_{1}, \ldots, P_{n}$ be a set of representatives of these $\Gamma$-conjugacy classes.

With respect to the fixed basepoint $x_{0}=K \in X=G / K$, each $P_{i}$ gives a horospherical decomposition of $X: X=N_{P_{i}} \times X_{P_{i}} \times A_{P_{i}}$. For every $r \gg 0$, the projection

$$
\Gamma_{P_{i}} \backslash N_{P_{i}} \times X_{P_{i}} \times A_{P_{i}, r} \rightarrow \Gamma \backslash X
$$


is an embedding, and $\Gamma \backslash X$ admits a disjoint decomposition

$$
\Gamma \backslash X=\omega_{0} \cup \coprod_{i=1}^{n} \Gamma_{P_{i}} \backslash N_{P_{i}} \times X_{P_{i}} \times A_{P_{i}, r},
$$

where $\omega_{0}=\omega_{0}(r)$ is a compact submanifold with boundary.

For a scattering geodesic $\gamma$ from the end of $P_{j}$ to the end of $P_{j}$, there exists $r_{0}(\gamma)$ such that for $r \geqslant r_{0}(\gamma)$, the intersection of $\gamma$ with $\Gamma_{P_{i}} \backslash N_{P_{i}} \times X_{P_{i}} \times A_{P_{i}, r}$ is a ray of the form $\gamma(t)=\left(n, m, e^{H_{0}+t H}\right)$, for some $n \in N_{P_{i}}, m \in X_{P_{i}}, H_{0} \in \mathfrak{a}_{P_{i}}, H \in \mathfrak{a}_{P_{i}}^{+}$, and the intersection with the end $\Gamma_{P_{j}} \backslash N_{P_{j}} \times X_{P_{j}} \times A_{P_{j}, r}$ is of a similar form.

Denote the boundary of the end $\Gamma_{P_{i}} \backslash N_{P_{i}} \times X_{P_{i}} \times A_{P_{i}, r}$ by $Y_{P_{i}, r}$. Then for $r>r_{0}(\gamma)$, the geodesic $\gamma(t)$ intersects each of $Y_{P_{i}, r}, Y_{P_{j}, r}$ at only one point, denoted by $\gamma\left(t_{2}(\gamma)\right), \gamma\left(t_{1}(\gamma)\right)$ respectively, and the length of the geodesic segment of $\gamma$ between $Y_{P_{i}, r}$ and $Y_{P_{j}, r}$ is equal to $t_{2}(\gamma)-t_{1}(\gamma)$. This length depends on $r$, but $t_{2}(\gamma)-t_{1}\left(\gamma_{1}\right)-2 r$ is canonical and is the sojourn time of $\gamma$.

The comments after Definition 2.7 in [4] was meant to convey this picture in an imprecise way. As pointed out by the reviewer, it should be emphasized that the height $r_{0}(\gamma)$ of the cusp neighborhoods depends on the geodesic $\gamma$, and when $\gamma$ varies, there is no uniform upper bound on $r_{0}(\gamma)$, and one cannot choose one fixed large $r$ or a fixed compact core $\omega_{0}(r)$ and define the sojourn times of all scattering geodesics this way. This unboundedness of $r_{0}(\gamma)$ can be seen clearly when $X$ is the Poincaré upper half plane.

On the other hand, for purposes in Section 4, Eq. (4.4), we need to fix a sufficiently large $r$ and define the sojourn time with respect to the fixed horocycles $Y_{P_{i}, r}, Y_{P_{j}, r}$. Then the correction definition is given in Definition 2.7, or formulated slightly differently by the reviewer.

To relate the sojourn time to the Bruhat decomposition, we need to lift the scattering geodesics to $X$. The disjoint decomposition $\Gamma \backslash X=\omega_{0}(r) \cup \coprod_{i=1}^{n} \Gamma_{P_{i}} \backslash N_{P_{i}} \times X_{P_{i}} \times A_{P, r}$ lifts a $\Gamma$-equivariant disjoint decomposition $X=X_{r} \cup \coprod_{P} N_{P} \times X_{P} \times A_{P, r(P)}$, where $X_{r}$ is a closed submanifold with $\Gamma \backslash X_{r}=\omega_{0}(r)$, and $P$ runs over all rational parabolic subgroups. For $P \in\left\{P_{1}, \ldots, P_{n}\right\}, r(P)=r$, and for other $P, r(P)$ is determined by the $\Gamma$-action on $X$. In fact, a formula for $r\left({ }^{\gamma} P_{i}\right), \gamma \in \Gamma$, is given in [5, p. 330]. Then a scattering geodesic $\gamma$ between the ends of $P_{i}, P_{j}$ is lifted to a geodesic $\tilde{\gamma}$ in $X$ which intersects the horospheres $N_{P_{i}} \times X_{P_{i}} \times \partial A_{P_{i}, r\left(P_{i}\right)}$ and $N_{\delta_{P_{j}}} \times X_{\delta_{P_{j}}} \times \partial A_{\delta_{P_{j}, r}\left({ }^{(} P_{j}\right)}$, for some $\delta \in \Gamma$, each at a unique point, and the distance between these two points along $\tilde{\gamma}$ is equal to $t_{2}(\gamma)-t_{1}(\gamma)$, the prenormalized sojourn time of $\gamma$. The lift $\tilde{\gamma}$ is also called a scattering geodesic between $P_{i},{ }^{\delta} P_{j}$, and its sojourn time is defined to be equal to that of $\gamma$.

Remark. - Since the formula for $r\left({ }^{\gamma} P\right)$ in [5, p. 330] is given in terms of the split component of $\gamma$ in the Langlands decomposition, it was misused in Proposition 2.10 and other places in [4] to conclude that the sojourn time is bounded from below up to a constant by the norm of this split component. Actually, the problem is that when $\gamma$ varies, the $N_{P_{i}}, X_{P_{i}}$ components of the intersection points of $\tilde{\gamma}$ with the horosphere are not necessarily bounded, and hence the desired bound is not true.

To prove this relation, we consider two cases: from one end to itself, and to another end. For the first case, fix one rational parabolic subgroup $P_{i}$. Then for any $\gamma \in \Gamma-\Gamma_{P_{i}}, P_{i},{ }^{\gamma} P_{i}$ are distinct. As proved in Proposition 2.10 in [4], there is a common split component of $P_{i}$ and ${ }^{\gamma} P_{i}$, and this common split component gives a smooth family of scattering geodesics in $X$ between $P_{i}$ and ${ }^{\gamma} P_{i}$ of the same sojourn time, which projects to a family from the end of $P_{i}$ to itself. As pointed out by the reviewer (see also [3, p. 120]), this sojourn time can be computed by the Bruhat decomposition of $\gamma$. 
Precisely, write $\gamma=u_{2} a m w u_{1}$, where $u_{1}, u_{2} \in N_{P_{i}}, a \in A_{P_{i}}, m \in M_{P_{1}}$, and $w \in K$ is a representative in the normalizer of $A_{P_{i}}$ of the nontrivial element of the Weyl group. Since ${ }^{\gamma} P_{i} \neq P_{i}, w$ is nontrivial.

PROPOSITION 2.1. - In the above notation, the sojourn time of the scattering geodesics between $P_{i}$ and ${ }^{\gamma} P_{i}$ is equal to $\|\log a\|$.

Proof. - Let $P^{\prime}={ }^{\gamma} P_{i}$. Then ${ }^{u_{2}^{-1}} P^{\prime}={ }^{a m w u_{1}} P_{i}={ }^{a m w} P_{i} \supset{ }^{a m w} A_{P_{i}}=A_{P_{i}}$. This implies that $P^{\prime} \supset{ }^{u_{2}} A_{P_{i}}$. Clearly, $P_{i} \supset{ }^{u_{2}} A_{P_{i}}$, and hence ${ }^{u_{2}} A_{P_{i}}$ is a common split component of $P^{\prime}$ and $P_{i}$.

It should be pointed out that the original split component $A_{P_{i}}$ is with respect to the basepoint $x_{0}$, i.e., stable under the Cartan involution associated with $x_{0}$, and the new split component ${ }^{u_{2}} A_{P_{i}}$ is with respect to the new basepoint $u_{2} x_{0}$. The Langlands decomposition of $P_{i}$ with respect to the basepoint $u_{2} x_{0}$ are $u_{2}$-conjugates of the decomposition $P_{i}=N_{P_{i}} M_{P_{i}} A_{P_{i}}$, and hence the family of scattering geodesics between $P_{i}, P^{\prime}$ is of the form $u_{2} e^{t H_{1}} m_{2} x_{0}$, where $m_{2} \in M_{P_{i}}, e^{H} \in A_{P_{i}}$, since $u_{2} e^{H} m_{2} x_{0}={ }^{u_{2}}\left(e^{H}\right){ }^{u_{2}} m_{2} u_{2} x_{0}$, and ${ }^{u_{2}}\left(e^{H}\right),{ }^{u_{2}} m_{2}$ are the Langlands coordinates with respect to the basepoint $u_{2} x_{0}$.

To compute the sojourn time, we need to determine the level of the horosphere which contains the point $\gamma^{-1}\left(u_{2} e^{H} m_{2} x_{0}\right)$, where $e^{H} \in \partial A_{P^{\prime}, r\left(P^{\prime}\right)}$, since the sojourn time can be computed by the $\Gamma$-equivariant decomposition of $X$ as mentioned above. By definition,

$$
\begin{aligned}
\gamma^{-1}\left(u_{2} e^{H} m_{2} x_{0}\right) & =u_{1}^{-1} w^{-1} m^{-1} a^{-1} u_{2}^{-1} u_{2} e^{H} m_{2} x_{0}=u_{1}^{-1} w^{-1} m^{-1} a^{-1} e^{H} m_{2} x_{0} \\
& =u_{1}^{-1} a e^{-H} m_{1} x_{0},
\end{aligned}
$$

where $m_{1}=w^{-1} m^{-1} m_{2} w$ is an element of $M_{P_{i}}$, and hence the $A_{P_{i}}$-component of $\gamma^{-1}\left(u_{2} e^{H} m_{2} x_{0}\right)$ is equal to $a e^{-H}$. This implies that the distance between the two horospheres of $P_{i}, P^{\prime}$ is equal to $\|\log a\|+2 r$, and hence the sojourn time of this family of scattering geodesics in $X$ is equal to $\|\log a\|$.

Fix a distinct pair of ends of $\Gamma \backslash X$ and the corresponding parabolic subgroups $P_{i}, P_{j}$. For any $\gamma \in \Gamma$, the pair $P_{j},{ }^{\gamma} P_{i}$ are still distinct. Choose $k \in K$ such that ${ }^{k} P_{i}=P_{j}$. Write the Bruhat decomposition $k^{-1} \gamma=u_{2} a m w u_{1}$ as above, or equivalently $\gamma=k u_{2} a m w u_{1}$, where $u_{1}, u_{2} \in N_{P_{i}}$, $a \in A_{P_{i}}, m \in M_{P_{i}}, w$ is the nontrivial element. Since the pair $\left(P_{j},{ }^{\gamma} P_{i}\right)=\left({ }^{k} P_{i},{ }^{\gamma} P_{i}\right)$ is conjugate to $\left(P_{i},{ }^{-1} \gamma P_{i}\right)$, by the same argument as above, we can prove the following result.

Proposition 2.2. - For a pair of distinct $\Gamma$-conjugacy classes $P_{i}, P_{j}$ and any $\gamma \in \Gamma$, the sojourn time of the family of scattering geodesics between $P_{j}$ and ${ }^{\gamma} P_{i}$ is equal to $\|\log a\|$ in the above notation.

Once we have identified the sojourn times with the norms $\|\log a\|$ as above, the second statement of Proposition 2.10 in [4] of discreteness of sojourn times follows from Lemma 6.1 in [3], which proves the discreteness of such norms $\|\log a\|$. Since the proof of Lemma 6.1 is complicated, we include a direct proof communicated to us by Hoffmann.

Proposition 2.3. - For every pair of ends of $\Gamma \backslash X$, not necessarily distinct, the set of sojourn times of families of scattering geodesics between them is discrete with finite multiplicity.

Proof. - For each rational parabolic subgroup $P$, let $C_{P, r}=N_{P} \times X_{P} \times A_{P, r}$. Then the $\varepsilon$-neighborhood of $C_{P, r}$ in $X$ is equal to $C_{P, r-\varepsilon}$. Since $P$ has $\mathbb{Q}$-rank 1 , there exists a compact subset $\omega \subset N_{P} \times X_{P}$ such that $\omega \Gamma_{P}=N_{P} \times X_{P}$, where $\Gamma_{P}=\Gamma \cap P$. By reduction theory, this implies that for any fixed $\varepsilon>0$, we can choose $r_{0}$ to be sufficiently large such that the sets $\delta C_{Q, r_{0}-\varepsilon}$ are disjoint, where $Q$ ranges over a set of representatives of $\Gamma$-conjugacy classes of rational parabolic subgroups, and for each $Q, \delta$ ranges over $\Gamma / \Gamma_{Q}$, and hence the mutual distances between $\delta C_{Q, r_{0}}$ are uniformly bounded below by $2 \varepsilon$. 
Now let $\gamma$ be a scattering geodesic in $X$ from ${ }^{\delta} Q$ to $P$ with sojourn time less than $t$. Then, for any $r \in \mathbb{R}, \gamma(\mathbb{R}) \subset C_{P,-t-r} \cup \delta C_{Q, r}$. However, $\gamma(\mathbb{R})$ is connected, so $C_{P,-t-r} \cap \delta C_{Q, r} \neq \emptyset$. Thus, if $N_{P Q}(t)$ denotes the number of scattering geodesics in $\Gamma \backslash X$ between the cusps corresponding to the $\Gamma$-conjugacy classes of $P$ and $Q$ of sojourn time less than $t$, then

$$
N_{P Q}(t) \leqslant \#\left\{\delta \in \Gamma \cap P \backslash \Gamma / \Gamma \cap Q \mid P \neq{ }^{\delta} Q, C_{P,-t-r} \cap \delta C_{Q, r} \neq \emptyset\right\} .
$$

We note that the double coset $\Gamma \cap P \backslash \Gamma / \Gamma \cap Q$ parametrizes the families of scattering geodesics between the ends of $\Gamma \backslash X$ corresponding to $Q$ and $P$. For any $r$, let $S_{P, r}=\omega \times A_{P, r}$ be a Siegel domain such that $(\Gamma \cap P) S_{P, r}=C_{P, r}$ and that the closure $S_{P, r_{1}, r_{2}}$ of $S_{P, r_{1}}-S_{P, r_{2}}$ is compact for $r_{1}<r_{2}$. If $C_{P,-t-r} \cap \delta C_{Q, r} \neq \emptyset$, we can find $\delta^{\prime} \in(\Gamma \cap P) \delta$ such that $S_{P,-t-r} \cap \delta^{\prime} C_{Q, r} \neq \emptyset$, and hence

$$
N_{P Q}(t) \leqslant \#\left\{\delta^{\prime} \in \Gamma / \Gamma \cap Q \mid P \neq{ }^{\delta^{\prime}} Q, S_{P,-t-r} \cap \delta^{\prime} C_{Q, r} \neq \emptyset\right\} .
$$

Let us specialize to $r=r_{0}$. By the choice of $r_{0}, S_{P, r_{0}} \cap \delta^{\prime} C_{Q, r_{0}}=\emptyset$ for all $\delta^{\prime} \in \Gamma, P \neq \delta^{\prime} Q$, and hence

$$
N_{P Q}(t) \leqslant \#\left\{\delta \in \Gamma / \Gamma \cap Q \mid P \neq{ }^{\delta} Q, S_{P,-t-r_{0}, r_{0}} \cap \delta C_{Q, r_{0}} \neq \emptyset\right\} .
$$

For every $\delta$ contributing to this sum we choose a point in $S_{P,-t-r_{0}, r_{0}} \cap \delta C_{Q, r_{0}}$. These points have mutual distance bounded away from zero, and by the compactness of $S_{P,-t-r_{0}, r_{0}}$ they must be finite in number. This proves that $N_{P Q}(t)$ is finite.

\section{Comments}

The generalization of Guillemin's formula in [3, Theorem 7.1] is called factorization of the scattering matrix, and is an important ingredient of the invariant trace formula in [3, Theorem 6.4]. The approach is quite algebraic, and the geometric structure of the scattering geodesics is not transparent in this formula.

On the other hand, the result in [4] is a generalization of Guillemin's formula by identifying geometrically the singular structures of the Fourier transform of the scattering matrix. In fact, such a relation between the periods of oscillation of the scattering matrix and the sojourn times is what Guillemin sought after in general [1, p. 75]. The dimension of the smooth families of the scattering geodesics is reflected in the size of the singularities, and the sojourn times determine the locations of the singularities. The different roles they play correspond to the factorization of the scattering matrix in [3] as a product of the Knapp-Stein intertwining operator and generalized Hecke operators. When we wrote [4], we were not aware of [3], or the relation between the sojourn time and the Bruhat decomposition in Propositions 2.1 and 2.2. In [1, p. 84, Eq. (B.2)], a formula for the unnormalized sojourn time is given. It can be shown that the formula for the normalized sojourn time is the same as the one given here in terms of the Bruhat decomposition. This relation between the sojourn time and the Bruhat decomposition in Propositions 2.1 and 2.2 gives more structure to the sojourn time.

\section{REFERENCES}

[1] Guillemin V., Sojourn times and asymptotic properties of the scattering matrix, Publ. RIMS, Kyoto Univ. 12 Suppl. (1977) 69-88.

[2] Hoffmann W., Review of [4] in Math. Review, AMS, 2002.

[3] Hoffmann W., An invariant trace formula for rank one lattices, Math. Nachr. 207 (1999) 93-131.

$4^{\mathrm{e}}$ SÉRIE - TOME $35-2002-\mathrm{N}^{\circ} 6$ 
[4] JI L., ZWORSKI M., Scattering matrices and scattering geodesics of locally symmetric spaces, Ann. Scient. Éc. Norm. Sup. 34 (2001) 441-469.

[5] Osborne M., WARner G., The Selberg trace formula II: partition, reduction, truncation, Pacific J. Math. 106 (1983) 307-496.

(Manuscrit reçu le 7 juin 2002; accepté, après révision, le 11 juillet 2002.)

\author{
Lizhen JI \\ Department of Mathematics, \\ University of Michigan, \\ Ann Arbor, MI 48109, USA \\ E-mail: 1ji@math.lsa.umich.edu \\ Maciej ZWORSKI \\ Mathematics Department, \\ University of California, \\ Berkeley, CA 94720, USA \\ E-mail: zworski@math.berkeley.edu
}

